\title{
Use of Healthcare Services Following Severe Hypoglycemia in Patients with Diabetes: Analysis of Real-World Data
}

Dalit Goldstein · Gabriel Chodick · Varda Shalev • Brian L. Thorsted ·

Lisa Elliott · Avraham Karasik

Received: January 13, 2016 / Published online: April 21, 2016

(C) The Author(s) 2016. This article is published with open access at Springerlink.com

\section{ABSTRACT}

Introduction: Severe hypoglycemia is a burden for both patients and the healthcare system payer alike. This study aimed to quantify the resource use associated with a severe hypoglycemic event (SHE) in patients with diabetes.

Methods: This retrospective cohort study compared resource use (e.g., physician visits, hospitalizations, and medications) 1 month pre- and post-SHE among patients with type 1 (T1D) or type 2 diabetes (T2D) from a large not-for-profit healthcare provider.

Results: From 2005 to 2014, 284 patients with T1D (52.5\% male, mean age 29.8 years, mean

Enhanced content To view enhanced content for this article go to http://www.medengine.com/Redeem/ A7B4F06002BC662B.

D. Goldstein · G. Chodick · V. Shalev

Maccabitech, Maccabi Healthcare Services, Tel Aviv, Israel

B. L. Thorsted · L. Elliott

Market Access, Novo Nordisk A/S, Søborg, Denmark

\section{A. Karasik ( $\square)$}

Department of Endocrinology, Sheba Medical

Center, Tel HaShomer, Ramat Gan, Israel

e-mail: karasik@post.tau.ac.il
$\mathrm{HbA}_{1 \mathrm{c}}$ 7.9\%) and 3691 patients with T2D $(47.6 \%$ male, mean age 67.1 years, mean $\mathrm{HbA}_{1 \mathrm{c}} 7.3 \%$ ) were eligible for inclusion in the study. In total, $95.4 \%$ of patients with T1D and $32.8 \%$ of patients with T2D were insulin treated, while $3.5 \%$ of patients with $\mathrm{T} 1 \mathrm{D}$ and $70.4 \%$ of patients with T2D were treated with oral drugs that could cause hypoglycemia (sulfonylureas or meglitinides). Hospital admissions increased by $95 \%$ in T1D and $127 \%$ in T2D $(P<0.001) 1$ month post-SHE versus 1 month pre-SHE. Of those admitted to hospital (T1D $n=59$; T2D $n=1214$ ), the mean length of stay was significantly longer during the month post- versus pre-SHE [2.08 vs. 0.88 days, $P=0.036$ (T1D) and 4.17 vs. 1.45 days, $P<0.001$ (T2D)]. Outpatient visits also increased by $37 \%$ for T1D and $47 \%$ for T2D between these two time periods $(P<0.001)$. The total monthly expense per patient increased by $46 \%$ and $87 \%$ for $\mathrm{T} 1 \mathrm{D}$ and $\mathrm{T} 2 \mathrm{D}$, respectively, from $\$ 485$ pre-SHE to $\$ 708$ post-SHE for T1D, and from $\$ 601$ pre-SHE to $\$ 1121$ post-SHE for T2D $(P<0.001)$. The greatest expense was hospital care, with increases of $179 \%$ and $166 \%$ for T1D and T2D, respectively, to $\$ 312$ and $\$ 706$ per patient/month. 
Conclusion: This real-world analysis from a large diabetes registry indicates an increased use of healthcare services, including more frequent and prolonged hospital admissions and outpatient visits after an SHE, which resulted in an increase in healthcare expense.

Funding: Novo Nordisk.

Keywords: Hospitalization; Hypoglycemia; Resource use

\section{INTRODUCTION}

Hypoglycemia is a common and serious side effect associated with glucose-lowering therapies. Hypoglycemia occurs frequently in insulin-treated patients, and prevalence is higher in patients with type 1 diabetes (T1D) than type 2 diabetes (T2D), but increases in frequency in T2D with disease duration as treatment intensifies [1-3]. Hypoglycemia is a major burden for the healthcare sector and society, due to the treatment cost and the associated lost productivity [4-6]; it is also a burden for patients, resulting in fear and anxiety, and disruption to their sleep, and their domestic and social life $[4,7,8]$. Moreover, hypoglycemia is associated with death, adverse cardiovascular outcomes, dementia, falls, and fractures [9]. Furthermore, hypoglycemia can occur suddenly and with varying severity [4, 9]. Severe hypoglycemic events (SHEs, requiring third-party assistance) may require hospitalization and inpatient care, leading to significant resource use and costs [10-12].

The aim of this study was to quantify the resource use and expense associated with an SHE in adult patients with diabetes, using the Maccabi Healthcare Services (MHS) database registry. The majority of studies investigating resource use following hypoglycemia provide retrospective data on resource use after an event, with many only including patients who report resource use. This study is unique in that data were collected before and after an SHE for a designated time period, enabling assessment of the change in resource use after an SHE. The time period under consideration for this analysis was 1 month pre-SHE and 1 month post-SHE.

\section{METHODS}

MHS is the second-largest Health Management Organization (HMO) in Israel, serving 25\% of the country's total population with approximately two million members. Since 1997, information on all members' interactions with MHS has been collected, including diagnoses, visits to primary and secondary care physicians, visits to outpatient clinics, hospitalizations, laboratory tests, and purchased and dispensed medications.

This observational, retrospective, non-interventional, cohort study analyzed de-identified patients' electronic medical record (EMR) data to compare resource use 1 month before and after the date that the index SHE was reported. The inclusion criteria for the MHS diabetes registry have been described previously [13, 14]. Briefly, the registry used criteria defined by the American Diabetes Association and included any patient with one or more of the following: $\mathrm{HbA}_{1 \mathrm{c}}$ $\geq 7.25 \%$, blood glucose $(\mathrm{BG}) \geq 11.1 \mathrm{mmol} / \mathrm{L}$, a preceding diagnosis of diabetes [identified using International Classification of Diseases version 9 (ICD-9) codes] together with a measurement of $\mathrm{HbA}_{1 \mathrm{c}} \geq 6.5 \%$ or $\mathrm{BG}>6.9 \mathrm{mmol} / \mathrm{L}$. The diabetes registry also included those who purchased $\geq 2$ monthly packs of 
anti-hyperglycemic medication during a 6-month period. The MHS diabetes registry was created for clinical purposes and its data are routinely updated by the patient's physicians, to maintain a very high level of validity. Patients were excluded if they were $<18$ years of age, were diagnosed with diabetes for $<12$ months, had $<12$ months' follow-up after their SHE, or if their type of diabetes was unknown. Data are presented for 1 month pre- and 1 month post-SHE. Data were also collected for 1 week and 12 months preand post-index date to conduct sensitivity analyses (data not shown).

Patients were included in this analysis if they had at least one SHE, identified by ICD-9 codes in the MHS registry between January 1, 2005 and April 30, 2013. Study index date (baseline) was defined as the earliest date of SHE diagnosis after a 12-month SHE-free period. This analysis considers data collected during 1 month before and after the SHE.

Data retrieved for this study included sociodemographic details, diabetes duration, body mass index (BMI), $\mathrm{HbA}_{1 \mathrm{c}}$, hypertension and cardiac comorbidities, and healthcare services utilization. Utilization data included hospitalizations, emergency department visits, outpatient visits and laboratory, pathology, radiology and imaging visits, medication, and purchases of self-measured blood glucose (SMBG) strips. The BMI value was the value taken nearest to the SHE (before or after it). $\mathrm{HbA}_{1 \mathrm{c}}$ value was the most recent result before the SHE. Socioeconomic status was based on residential socioeconomic index, ranging from 1 (lowest) to 20 (highest). In the patient records where diabetes type was not recorded, a classification of T1D was made if the patient was using insulin or had a positive glutamic acid decarboxylase (GAD) or islet cell antibody, and was under the age of 40 years. A classification of T2D was made if the patient was not using insulin for at least 1.5 years or had a positive C-peptide test. In other cases, the diabetes type was classified as unknown and the patient was excluded from the analysis.

Costs of different healthcare services were taken from the Israeli Ministry of Health price list in Israeli new shekels and were converted to \$US dollars. Costs per patient represent mean values for all patients included in the study. The study was approved by MHS' ethics committee and performed in accordance with the Declaration of Helsinki [15]. The study was performed according to the STrengthening the Reporting of OBservational studies in Epidemiology (STROBE) Statement for cohort studies [16].

\section{Statistical Analysis}

Resource utilization and healthcare costs were compared in the time period ( 1 month) before and after index SHE. Continuous variables (length of hospital stay and healthcare expenditure) were compared using a paired Student's $t$ test. Chi square (number of hospitalizations) and McNemar's tests (hospital visits comparison) were used to compare categorical variables. Wilcoxon signed-rank test was used to compare ordinal variables (the number of hospitalizations per patient and expense comparisons). A Kruskal-Wallis test was used to compare between-group differences following stratification by BMI and socioeconomic status, and a Mann-Whitney test was used to compare insulin-treated versus non-insulin-treated groups. Statistical significance was set at $P<0.05$. All analyses were conducted using IBM SPSS software (IBM SPSS Statistics for Windows, version 21.0, released 2012, IBM Corporation, Armonk, NY, USA). 


\section{RESULTS}

\section{Study Population}

A total of 3975 patients were eligible for inclusion in the study; their characteristics at index/time of SHE are summarized in Table 1. Overall, $7.1 \%$ of patients in the study were categorized as T1D $(n=284)$, and $92.9 \%$ as T2D ( $n=3691)$. At index, $95.4 \%$ of patients with T1D and $32.8 \%$ of patients with T2D were insulin treated, $3.5 \%$ with $\mathrm{T} 1 \mathrm{D}$ and $70.4 \%$ with T2D were treated with oral antidiabetic drugs that could cause hypoglycemia (sulfonylureas or meglitinides), and $7.7 \%$ with T1D and $76.4 \%$ with T2D used oral drugs known to cause hypoglycemia only rarely (metformin, gliptins, glitazones, and acarbose). During 9 years of the study, there were only slight and insignificant differences in the number of SHEs reported by patients with diabetes in the registry each year.

\section{Hospital Visits}

In the month following an SHE, 25\% $(n=992)$ of the study population were hospitalized and $10 \% \quad(n=401)$ visited the emergency

Table 1 Study population characteristics

\begin{tabular}{|c|c|c|}
\hline Characteristics & T1D & T2D \\
\hline$n$ & 284 & 3691 \\
\hline Age, years & $29.8 \pm 6.7$ & $67.1 \pm 11.7$ \\
\hline Males, $n(\%)$ & $149(52.5)$ & $1756(47.6)$ \\
\hline \multicolumn{3}{|l|}{ Duration of diabetes, years, $n(\%)$} \\
\hline$<5$ & $97(34.2)$ & $938(25.4)$ \\
\hline $5-9$ & $131(46.1)$ & $1824(49.4)$ \\
\hline $10+$ & $56(19.7)$ & $929(25.2)$ \\
\hline BMI, $\mathrm{kg} / \mathrm{m}^{2}$ & $25.5 \pm 4.6$ & $29.9 \pm 5.6$ \\
\hline $\mathrm{HbA}_{1 \mathrm{c}}, \%$ (most recent prior to $\mathrm{SHE}$ ) & $7.9 \pm 1.8$ & $7.3 \pm 1.5$ \\
\hline Cardiac comorbidity-yes, $n$ (\%) & $14(4.9)$ & $1653(44.8)$ \\
\hline Hypertension comorbidity-yes, $n$ (\%) & $25(8.8)$ & $2914(78.9)$ \\
\hline Hospitalization a week around index date, $n(\%)$ & $47(16.5)$ & $973(26.4)$ \\
\hline Socioeconomic status ${ }^{a}$ & $11.1 \pm 4.2$ & $11.3 \pm 4.2$ \\
\hline Fractures 1 month around SHE, $n(\%)$ & $3(1.1)$ & $79(2.1)$ \\
\hline \multicolumn{3}{|l|}{ Additional SHE within 12 months of index, $n$ (\%) } \\
\hline 1 & $52(18.3)$ & $661(17.9)$ \\
\hline 2 & $16(5.6)$ & $173(4.7)$ \\
\hline 3 & $16(5.7)$ & $159(4.3)$ \\
\hline
\end{tabular}

Data are mean \pm standard deviation, unless otherwise specified

$B M I$ body mass index, $S H E$ severe hypoglycemic event, $T I D$ type 1 diabetes, $T 2 D$ type 2 diabetes

${ }^{a}$ On a scale from 1 (lowest) to 20 (highest) 
Table 2 Number of hospitalization episodes

\begin{tabular}{lccccr}
\hline & $\begin{array}{l}\text { Total no. of } \\
\text { hospitalizations }\end{array}$ & $\begin{array}{l}\text { 1 month } \\
\text { pre-SHE }\end{array}$ & $\begin{array}{l}\text { 1 month } \\
\text { post-SHE }\end{array}$ & Change (\%) & P value \\
\hline T1D & & & & & \\
Total & 68 & 23 & 45 & 95 & $<0.001$ \\
Non-surgical & 65 & 22 & 43 & 95 & $<0.001$ \\
Surgical & 3 & 1 & 2 & 100 & 0.861 \\
T2D & & & & 127 & $<0.001$ \\
Total & 1598 & 488 & 1110 & 139 & $<0.001$ \\
Non-surgical & 1460 & 430 & 1030 & 37 & 0.075 \\
Surgical & 138 & 58 & 80 & & \\
\hline
\end{tabular}

Total number of hospitalizations divided according to type of procedure (non-surgical or surgical) and diabetes type. Analysis conducted using a Chi-square test

SHE severe hypoglycemic event, TID type 1 diabetes, T2D type 2 diabetes

department. The number of hospital admissions increased significantly (by 95\% for T1D and by $127 \%$ for T2D, $P<0.001)$ in the month following the SHE (Table 2) compared with the month before the SHE. Additionally, the proportion of patients with 1, 2, or 3+ hospital admissions also increased significantly during this period versus the previous month (Fig. 1). The proportion of patients with T1D hospitalized on one occasion increased 1 month post-SHE but there was no increase in the number of patients requiring two or three hospitalizations. In patients with $\mathrm{T} 2 \mathrm{D}$, there was an increase in the proportion of patients with 1,2 , and $3+$ hospitalizations 1 month post- versus 1 month pre-SHE.

Of the patients admitted to hospital preand/or post-SHE (T1D: $n=59,1.4 \%$; T2D: $n=1214,30.5 \%$ ), the overall mean duration of stay was significantly longer during the month post- versus pre-SHE in both patients with T1D and T2D (T1D: 2.08 vs. 0.88 days, $P=0.036$; T2D: 4.17 vs. 1.45 days, $P<0.001$; Table 3 ). The mean increase in hospital stay post-SHE was longer in patients with T1D and T2D admitted for non-surgical procedures and patients with T2D admitted for surgical procedures, but not patients with T1D admitted for surgical procedures (Table 3 ). Of the 1273 patients who were hospitalized in the month pre- or post-SHE, $148 \quad(3.7 \%$ of the total study population; $11.6 \%$ of the hospitalized patients) were hospitalized both in the month pre- and the month post-SHE. The mean duration of stay in patients with T2D hospitalized pre- and post-SHE was significantly longer in the month post-SHE compared with the month pre-SHE $(9.36$ vs. 5.84 days, $P=0.002)$, whereas there was no significant difference in those with T1D (Table 3).

In the month following an SHE, the total number of outpatient visits increased significantly from 744 to 1022 (37\% increase, $P<0.001)$ in patients with T1D, and from 10,626 to 15,685 (47\% increase, $P<0.001)$ in patients with T2D. The greatest increases were in visits to endocrinologists, nurses, and dieticians (Table 4). There was a non-significant increase in outpatient pathology, radiology, and imaging visits, and a significant increase in the number of 

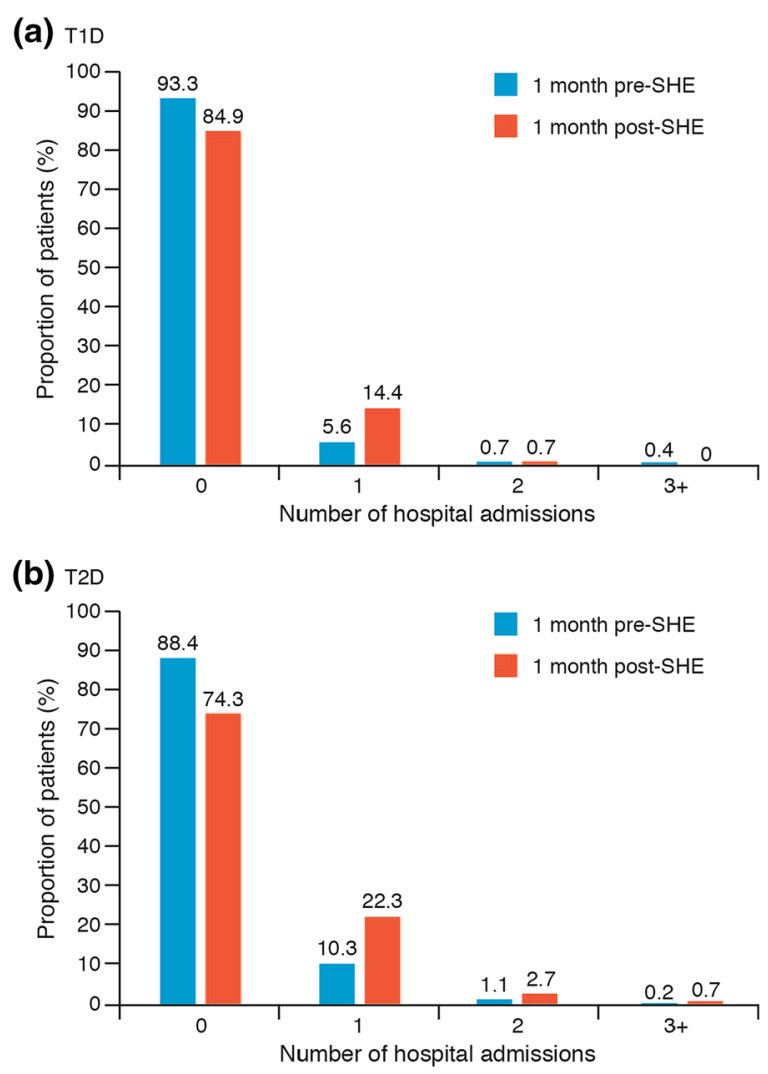

Fig. 1 Proportion of patients with a T1D and b T2D who had hospital admissions 1 month pre-SHE versus 1 month post-SHE. a $P<0.009$; b $P<0.001$ for comparison of number of hospitalizations pre-SHE versus post-SHE (Wilcoxon test). SHE severe hypoglycemic event, $T 1 D$ type 1 diabetes, $T 2 D$ type 2 diabetes

lab tests conducted following an SHE in patients with T2D (Table 4). There was also a significant increase in the number of medication purchases post-SHE, including a $61 \%$ increase in SMBG sticks purchases in patients with T2D $(P<0.001)$ and a $20 \%$ increase in diabetic medication purchases in patients with T1D $(P=0.014$; Table 4).

\section{Cost of Treatment}

The increase in frequency and duration of hospitalization and the frequency of outpatient visits resulted in a significant increase in the total costs, hospital costs, and outpatient costs in the month following an SHE compared with the month before (Fig. 2). The total expense per patient per month increased by $46 \%(P=0.001)$ from $\$ 485$ to $\$ 708$ for T1D and $87 \%(P<0.001)$ from $\$ 601$ pre-SHE to $\$ 1121$ post-SHE for T2D. The greatest expense, and the greatest increase in expense, was hospitalization, with an increase per patient per month from $\$ 112$ to $\$ 312(+179 \%$, $P=0.003)$ in $\mathrm{T} 1 \mathrm{D}$, and from $\$ 265$ to $\$ 706$ $(+166 \%, P<0.001)$ in T2D. Additionally, there was a significant increased expenditure of $37 \%$ in T1D and $46 \%$ in T2D for visits to healthcare specialists, and $34 \%$ in T2D for emergency department visits in the month following an SHE compared with the month pre-SHE (Fig. 2). In this analysis, there was no significant change in the expenses associated with pathology, radiology, and imaging or lab tests.

As additional sensitivity analyses, healthcare expenses were also stratified by BMI, socioeconomic index, whether or not the patient was insulin treated, Charlson comorbidity index, and $\mathrm{HbA}_{1 \mathrm{c}}$ (Table 5). There were no significant differences in expenses across BMI categories, socioeconomic status, $\mathrm{HbA}_{1 \mathrm{c}}$ levels, or insulin treatment in both patients with T1D and T2D. In patients with $\mathrm{T} 2 \mathrm{D}$, there was a significant increase in expenses for Charlson index categories, with patients with Charlson index of +4 having a $91 \%$ increase in mean expenses per patient in the month post-SHE versus pre-SHE. No significant differences for Charlson index categories were detected for patients with T1D (Table 5).

\section{DISCUSSION}

In this real-world, non-interventional analysis of data from a large-scale diabetes registry, severe hypoglycemia was associated with 
Table 3 Mean duration (days) of hospital stay per patient 1 month pre- and 1 month post-SHE

\begin{tabular}{|c|c|c|c|c|c|}
\hline & Number of patients & 1 month pre-SHE & 1 month post-SHE & Change (\%) & $P$ value \\
\hline \multicolumn{6}{|c|}{ All hospitalized patients } \\
\hline \multicolumn{6}{|l|}{ T1D } \\
\hline Total & 59 & $0.88 \pm 2.32$ & $2.08 \pm 3.45$ & 136 & 0.036 \\
\hline Non-surgical & $57^{\mathrm{a}}$ & $0.91 \pm 2.36$ & $2.21 \pm 3.46$ & 142 & 0.041 \\
\hline Surgical & $3^{\mathrm{a}}$ & 0.00 & $0.66 \pm 1.15$ & - & 0.423 \\
\hline \multicolumn{6}{|l|}{$\mathrm{T} 2 \mathrm{D}$} \\
\hline Total & 1214 & $1.45 \pm 3.28$ & $4.17 \pm 8.40$ & 187 & $<0.001$ \\
\hline Non-surgical & $1146^{a}$ & $1.29 \pm 2.71$ & $3.95 \pm 7.68$ & 206 & $<0.001$ \\
\hline Surgical & $138^{\mathrm{a}}$ & $2.07 \pm 5.04$ & $3.85 \pm 7.97$ & 85 & 0.044 \\
\hline \multicolumn{6}{|c|}{ Patients hospitalized both pre- and post-SHE } \\
\hline T1D & 2 & $5.50 \pm 0.70$ & $7.00 \pm 7.07$ & 27 & 0.830 \\
\hline $\mathrm{T} 2 \mathrm{D}$ & 146 & $5.84 \pm 5.71$ & $9.36 \pm 13.29$ & 60 & 0.002 \\
\hline
\end{tabular}

Data are mean \pm standard deviation for hospitalized patients only. $P$ values are based on a paired $t$ test, which assumes independence between groups

SHE severe hypoglycemic event, TID type 1 diabetes, T2D type 2 diabetes

a Some patients had both surgical and non-surgical procedures during the time period assessed

increased use of healthcare services and resources, resulting in a significant increase in healthcare expenses in the month following an SHE compared with the month pre-SHE. These results indicate that in the month following an SHE, patients have significantly more frequent and prolonged hospital admissions and outpatient visits versus the month pre-SHE. While it is recognized that factors other than the SHE could influence the data over the time period, the non-significant change in pathology, radiology, and imaging visits would suggest that there was no change in concomitant serious illnesses.

The increase in the proportion of patients with 1,2 , and $3+$ hospitalizations and the increase in length of hospital stay 1 month post- versus 1 month pre-SHE were higher for patients with T2D compared with T1D, possibly due to the higher rate of comorbidities and greater age in these populations. It is possible that patients with T1D and their carers may also have more experience at coping with SHEs, and therefore be less inclined to go to the hospital, compared with patients with T2D who may not be insulin treated or have been treated with insulin for a shorter duration, and therefore have experienced fewer SHEs. It should also be noted that the number of patients in the T1D category was smaller than in the T2D category, and this may partly explain why the results did not reach significance. However, other studies have also shown that hospitalization for severe hypoglycemia is more frequent in patients with T2D compared with T1D [10, 17]. These include a study of the resource use associated with severe hypoglycemia in insulin-treated patients with diabetes from a large clinical trial program, which showed that severe hypoglycemia often results in the use of emergency services or 
Table 4 Number of outpatient visits, tests and medication pack purchases 1 month pre- and 1 month post-SHE

\begin{tabular}{|c|c|c|c|c|}
\hline & 1 month pre-SHE & 1 month post-SHE & Change (\%) & $P$ value \\
\hline \multicolumn{5}{|l|}{ Outpatient visits } \\
\hline \multicolumn{5}{|l|}{$\mathrm{T} 1 \mathrm{D}$} \\
\hline Endocrinology & 78 & 147 & 88 & $<0.001$ \\
\hline Nursing care & 52 & 79 & 51 & 0.023 \\
\hline Dietician & 42 & 71 & 69 & 0.008 \\
\hline Mental care & 13 & 24 & 84 & 0.100 \\
\hline General medicine & 359 & 534 & 48 & $<0.001$ \\
\hline Physiotherapy & 17 & 18 & 5 & 1.000 \\
\hline Cardiology & 4 & 1 & -75 & 0.375 \\
\hline Internal medicine & 89 & 95 & 6 & 0.712 \\
\hline Ophthalmology & 40 & 23 & -42 & 0.044 \\
\hline Surgery & 13 & 2 & -84 & 0.007 \\
\hline Orthopedics & 37 & 28 & -24 & 0.321 \\
\hline Total & 744 & 1022 & 37 & $<0.001$ \\
\hline \multicolumn{5}{|l|}{$\mathrm{T} 2 \mathrm{D}$} \\
\hline Endocrinology & 346 & 960 & 177 & $<0.001$ \\
\hline Nursing care & 747 & 1287 & 72 & $<0.001$ \\
\hline Dietician & 341 & 556 & 63 & $<0.001$ \\
\hline Mental care & 210 & 307 & 46 & $<0.001$ \\
\hline General medicine & 6079 & 9326 & 53 & $<0.001$ \\
\hline Physiotherapy & 595 & 747 & 25 & $<0.001$ \\
\hline Cardiology & 148 & 185 & 25 & 0.049 \\
\hline Internal medicine & 832 & 962 & 15 & 0.002 \\
\hline Ophthalmology & 506 & 556 & 9 & 0.133 \\
\hline Surgery & 367 & 376 & 2 & 0.769 \\
\hline Orthopedics & 455 & 423 & -7 & 0.295 \\
\hline Total & 10,626 & 15,685 & 47 & $<0.001$ \\
\hline \multicolumn{5}{|l|}{ Outpatient tests } \\
\hline \multicolumn{5}{|l|}{$\mathrm{T} 1 \mathrm{D}$} \\
\hline Pathology, radiology and imaging & 9 & 4 & -125 & 0.166 \\
\hline Lab tests (all) & 122 & 110 & -9 & 0.470 \\
\hline $\mathrm{HbA}_{1 \mathrm{c}}$ tests & 83 & 68 & -18 & 0.225 \\
\hline
\end{tabular}


Table 4 continued

\begin{tabular}{|c|c|c|c|c|}
\hline & 1 month pre-SHE & 1 month post-SHE & Change (\%) & $P$ value \\
\hline \multicolumn{5}{|l|}{$\mathrm{T} 2 \mathrm{D}$} \\
\hline Pathology, radiology and imaging & 203 & 240 & 15 & 0.073 \\
\hline Lab tests (all) & 2221 & 2590 & 16 & $<0.001$ \\
\hline $\mathrm{HbA}_{1 \mathrm{c}}$ tests & 1019 & 1117 & 9 & 0.036 \\
\hline \multicolumn{5}{|l|}{ Medication packs } \\
\hline \multicolumn{5}{|l|}{$\mathrm{T} 1 \mathrm{D}$} \\
\hline Total medication & 1170 & 1313 & 12 & 0.045 \\
\hline Diabetic medication & 493 & 594 & 20 & 0.014 \\
\hline SMBG sticks & 492 & 584 & 18 & 0.091 \\
\hline \multicolumn{5}{|l|}{$\mathrm{T} 2 \mathrm{D}$} \\
\hline Total medication & 40,711 & 44,895 & 10 & $<0.001$ \\
\hline Diabetic medication & 8494 & 8893 & 4 & 0.069 \\
\hline SMBG sticks & 1774 & 2864 & 61 & $<0.001$ \\
\hline
\end{tabular}

Outpatient visits and outpatient tests comparisons were conducted using a McNemar's test. Medication packs comparisons were conducted using a Wilcoxon test

$S H E$ severe hypoglycemic event, $S M B G$ self-measured blood glucose, $T 1 D$ type 1 diabetes, $T 2 D$ type 2 diabetes

ambulance calls and in hospital treatment [17]. Severe hypoglycemia is recognized as a common cause of hospitalization in elderly patients with diabetes and those with comorbidities [18, 19], and in one study accounted for $94.6 \%$ of all endocrine emergency hospitalizations in older patients [20].

The results presented here are consistent with those from several other studies reporting the extensive resource use and cost implications of severe hypoglycemia for healthcare providers/payers. Although the costs vary depending on the countries included and their healthcare systems [6, $10-12,17,21]$, together they illustrate that hypoglycemia is a significant burden and that efforts to minimize it are likely to reduce the economic burden of diabetes.
Previous studies into hypoglycemia-related resource use did not compare the before and after costs for a specific population of patients. This study benefits from the pre- and post-event design, allowing for elucidation of the change in resource use and expenses to be compared, while correcting for background resource use. In support of the robustness of these results, sensitivity analyses conducted with 1 week and 1 year pre- and post-SHE data showed similar results (data not shown). The study also benefits from the level of detail captured and the size of the population included in the registry. Following the 1994 Israel National Health Act, MHS may not deny coverage to applicants on any grounds, including age or state of health, and thus these results are a representation of all sectors of the Israeli population, except for young adults aged 18-21 years, due to the 

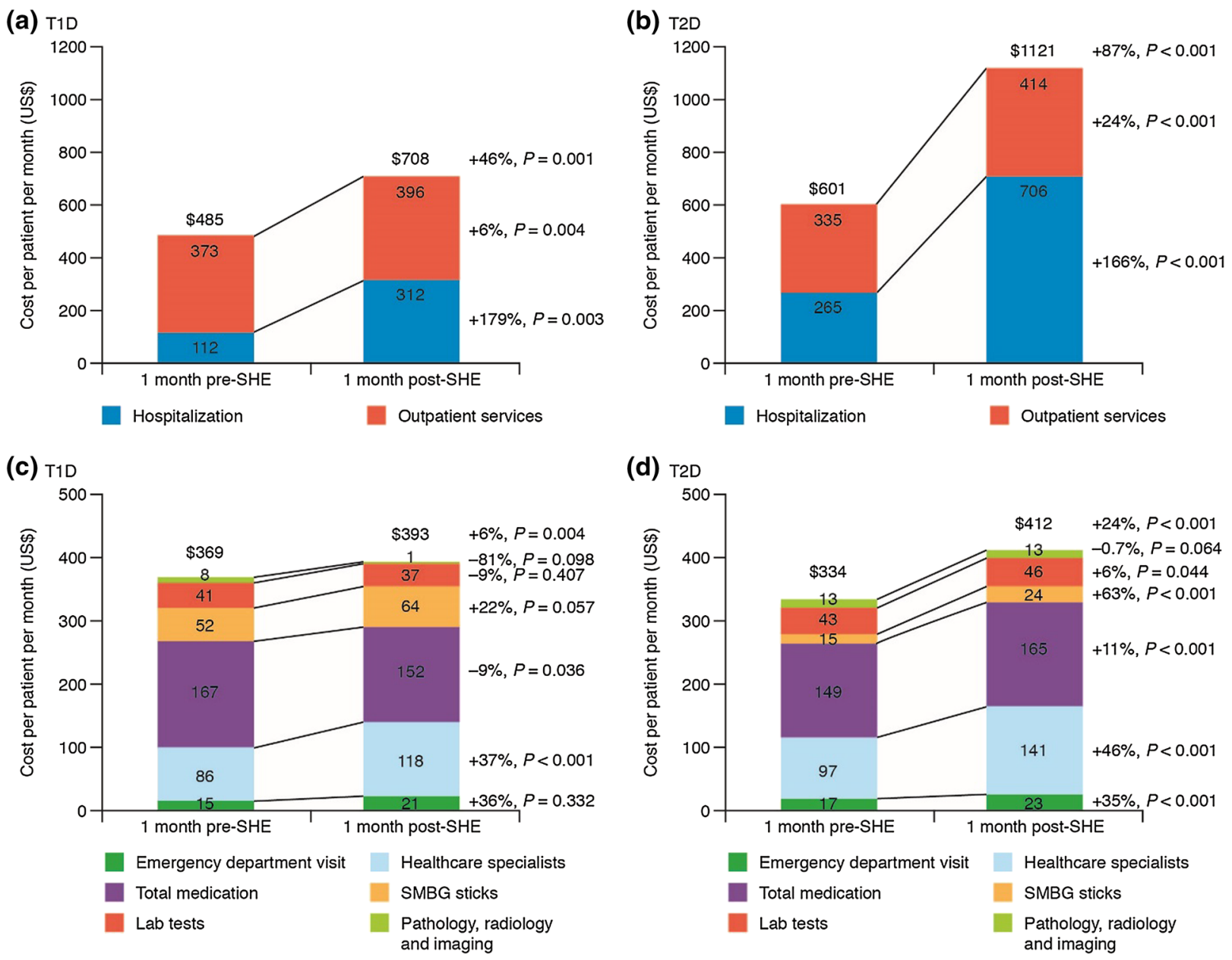

Fig. 2 Healthcare costs per patient 1 month pre- and 1 month post-SHE total costs for a T1D and $\mathbf{b}$ T2D, and breakdown of outpatient costs for $\mathbf{c}$ T1D and d T2D. Cost

high percentage of them being enlisted in the Israeli Defense Forces.

A limitation of this study is that the registry did not record the cause of SHEs or the reason why patients were hospitalized, so the primary reason for hospitalization may not have been related to diabetes or hypoglycemia in all cases. Some patients were hospitalized pre-SHE; however, in the patients who were hospitalized both pre- and post-SHE, the duration of stay was significantly increased post-SHE, so experiencing an SHE may have contributed to their longer hospital stay. The number of patients with T1D

(d) $\mathrm{T} 2 \mathrm{D}$

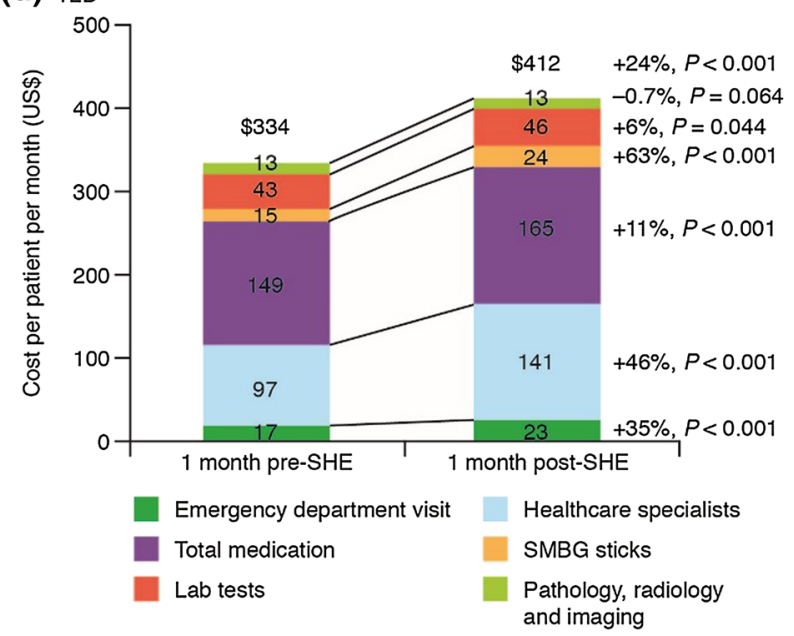

comparisons conducted using Wilcoxon test. $S H E$ severe hypoglycemic event, $S M B G$ self-measured blood glucose, $T 1 D$ type 1 diabetes, $T 2 D$ type 2 diabetes

included in the analysis was low, and they may not be considered a representative sample of patients with T1D because of their age ( $>18$ years) and late diagnosis age (around the age of 20 years). It is also not known how many non-severe hypoglycemia events the study population experienced during the designated time period, or whether they contributed to the increase in resource use observed here; nonetheless, this should not affect the conclusion of the study. Furthermore, the patients' diabetes-related complications may also have contributed to resource use. 
Table 5 Total expenses per patient stratified by BMI, socioeconomic status, and insulin use

\begin{tabular}{|c|c|c|c|c|c|c|}
\hline & & \multicolumn{2}{|c|}{ Costs US \$ } & \multicolumn{2}{|c|}{ Pre-SHE vs. post-SHE } & \multirow{2}{*}{$\begin{array}{l}\text { Between-group } \\
\text { comparison }\end{array}$} \\
\hline & & $\begin{array}{l}1 \text { month } \\
\text { pre-SHE }\end{array}$ & $\begin{array}{l}1 \text { month } \\
\text { post-SHE }\end{array}$ & $\begin{array}{l}\text { Change } \\
(\%)\end{array}$ & $P$ value $^{b}$ & \\
\hline \multicolumn{7}{|l|}{ T1D } \\
\hline \multirow[t]{3}{*}{ BMI, kg/m² $(n)$} & $<25(172)$ & 441 & 740 & 67 & 0.004 & $0.865^{c}$ \\
\hline & $26-30(80)$ & 571 & 677 & 18 & 0.173 & \\
\hline & $>31(30)$ & 531 & 646 & 21 & 0.192 & \\
\hline \multirow{3}{*}{ Socioeconomic status ${ }^{\mathrm{a}}(n)$} & Low (37) & 638 & 708 & 10 & 0.839 & $0.680^{c}$ \\
\hline & Medium (115) & 596 & 769 & 29 & 0.051 & \\
\hline & High (72) & 333 & 656 & 96 & 0.037 & \\
\hline \multirow{2}{*}{ Insulin use $(n)$} & Yes $(271)$ & 494 & 677 & 36 & 0.001 & $0.883^{\mathrm{d}}$ \\
\hline & No (13) & 298 & 1366 & 357 & 0.650 & \\
\hline \multirow{3}{*}{$\begin{array}{l}\text { Charlson comorbidity } \\
\text { index }^{\mathrm{e}}(n)\end{array}$} & Low (166) & 448 & 612 & 36 & 0.008 & $0.385^{\mathrm{c}}$ \\
\hline & Medium (88) & 431 & 730 & 69 & 0.212 & \\
\hline & High (30) & 849 & 1177 & 38 & 0.072 & \\
\hline \multirow[t]{3}{*}{$\mathrm{HbA}_{1 \mathrm{c}}(n)$} & $\leq 7 \%(99)$ & 538 & 776 & 44 & 0.061 & $0.984^{\mathrm{c}}$ \\
\hline & $7-9 \%(122)$ & 420 & 541 & 28 & 0.013 & \\
\hline & $>9 \%(61)$ & 530 & 899 & 69 & 0.227 & \\
\hline \multicolumn{7}{|l|}{$\mathrm{T} 2 \mathrm{D}$} \\
\hline \multirow[t]{3}{*}{ BMI, $\mathrm{kg} / \mathrm{m}^{2}(n)$} & $<25(908)$ & 652 & 1271 & 94 & $<0.001$ & $0.574^{\mathrm{c}}$ \\
\hline & $26-30(1311)$ & 538 & 990 & 83 & $<0.001$ & \\
\hline & $>31(1358)$ & 564 & 1007 & 78 & $<0.001$ & \\
\hline \multirow[t]{3}{*}{ Socioeconomic status $^{\mathrm{a}}(n)$} & Low (563) & 590 & 955 & 61 & $<0.001$ & $0.528^{c}$ \\
\hline & Medium (1600) & 603 & 1187 & 96 & $<0.001$ & \\
\hline & High (1057) & 589 & 1139 & 93 & $<0.001$ & \\
\hline \multirow[t]{2}{*}{ Insulin use $(n)$} & Yes (1210) & 687 & 1363 & 98 & $<0.001$ & $0.187^{\mathrm{d}}$ \\
\hline & No (2481) & 558 & 1003 & 79 & $<0.001$ & \\
\hline \multirow{3}{*}{$\begin{array}{l}\text { Charlson comorbidity } \\
\text { index }^{\mathrm{e}}(n)\end{array}$} & Low (776) & 315 & 552 & 75 & $<0.001$ & $<0.001^{\mathrm{c}}$ \\
\hline & Medium (1056) & 483 & 865 & 78 & $<0.001$ & \\
\hline & High (1859) & 787 & 1504 & 91 & $<0.001$ & \\
\hline
\end{tabular}


Table 5 continued

\begin{tabular}{|c|c|c|c|c|c|c|}
\hline & & \multicolumn{2}{|c|}{ Costs US $\$$} & \multicolumn{2}{|c|}{ Pre-SHE vs. post-SHE } & \multirow{2}{*}{$\begin{array}{l}\text { Between-group } \\
\text { comparison }\end{array}$} \\
\hline & & $\begin{array}{l}1 \text { month } \\
\text { pre-SHE }\end{array}$ & $\begin{array}{l}1 \text { month } \\
\text { post-SHE }\end{array}$ & $\begin{array}{l}\text { Change } \\
(\%)\end{array}$ & $P$ value ${ }^{b}$ & \\
\hline \multirow[t]{3}{*}{$\mathrm{HbA}_{1 \mathrm{c}}(n)$} & $\leq 7 \%(1870)$ & 600 & 1156 & 92 & $<0.001$ & $0.882^{c}$ \\
\hline & $7-9 \%(1382)$ & 568 & 1036 & 82 & $<0.001$ & \\
\hline & $>9 \%(436)$ & 712 & 1243 & 74 & $<0.001$ & \\
\hline
\end{tabular}

$B M I$ body mass index, SHE severe hypoglycemic event, TID type 1 diabetes, T2D type 2 diabetes

a Socioeconomic status: Residential socioeconomic index ranging from 1 (lowest) to 20 (highest); low 1-6; medium 7-13; high 14-20

b Statistical analysis based on a Wilcoxon signed-rank test

c Statistical analysis based on a Kruskal-Wallis test

d Statistical analysis based on a Mann-Whitney test

e Charlson comorbidity index: comorbidity index ranging from 1 (lowest) to 16 (highest); low $\leq 1$; medium 2-3; high $\geq 4$

\section{CONCLUSIONS}

In conclusion, this real-world analysis of nearly 4000 patients with diabetes who experienced an SHE documents the healthcare resources used, and expenses associated with severe hypoglycemia. The frequency and length of hospital stay increased, the number of outpatient visits increased, and the cost of treatment increased in the month post-SHE compared with the month pre-SHE.

\section{ACKNOWLEDGMENTS}

This study and the associated article processing charges for publication were funded by Novo Nordisk. All named authors meet the International Committee of Medical Journal Editors (ICMJE) criteria for authorship for this manuscript, take responsibility for the integrity of the work as a whole, and have given final approval to the version to be published. The authors take full responsibility for the content of the manuscript but are grateful to Adele Buss PhD and Daria Renshaw from Watermeadow Medical, an Ashfield Company, for medical writing and editing/submission support. This support was funded by Novo Nordisk A/S. Parts of this study have been published as abstracts and posters at the American Diabetes Association, 75th Annual Scientific Sessions, June 5-9, 2015, Boston, MA, USA and the 51st Annual Meeting of the European Association for the Study of Diabetes (EASD), September 14-18, 2015, Stockholm, Sweden.

Disclosures. Dalit Goldstein has no financial disclosures to declare. Gabriel Chodick has received research support from Merck, AbbVie, Pfizer, and Medial Cancer Research. Varda Shalev has received research support from Maccabitech. Brian L. Thorsted is an employee and shareholder of Novo Nordisk A/S. Lisa Elliott is an employee of Novo Nordisk A/S. Avraham Karasik has served on advisory panels for Novo Nordisk A/S and Merck Sharp and Dohme, and as a consultant for, and received research support from, Novo Nordisk A/S, Merck Sharp and Dohme, and Boehringer-Ingelheim.

Compliance with Ethics Guidelines. All procedures followed were in accordance with 
the ethical standards of the responsible committee on human experimentation (institutional and national) and with the Helsinki Declaration of 1964, as revised in 2013. Informed consent was not required for this study.

Open Access. This article is distributed under the terms of the Creative Commons Attribution-NonCommercial 4.0 International License (http://creativecommons.org/licenses/ by-nc/4.0/), which permits any noncommercial use, distribution, and reproduction in any medium, provided you give appropriate credit to the original author(s) and the source, provide a link to the Creative Commons license, and indicate if changes were made.

\section{REFERENCES}

1. Akram K, Pedersen-Bjergaard U, Carstensen B, Borch-Johnsen K, Thorsteinsson B. Frequency and risk factors of severe hypoglycaemia in insulin-treated type 2 diabetes: a cross-sectional survey. Diabet Med. 2006;23:750-6.

2. Donnelly LA, Morris AD, Frier BM, et al. Frequency and predictors of hypoglycaemia in type 1 and insulin-treated type 2 diabetes: a population-based study. Diabet Med. 2005;22:749-55.

3. UK Hypoglycaemia Study Group. Risk of hypoglycaemia in types 1 and 2 diabetes: effects of treatment modalities and their duration. Diabetologia. 2007;50:1140-7.

4. Brod M, Christensen T, Thomsen TL, Bushnell DM. The impact of non-severe hypoglycemic events on work productivity and diabetes management. Value Health. 2011;14:665-71.

5. Jonsson L, Bolinder B, Lundkvist J. Cost of hypoglycemia in patients with type 2 diabetes in Sweden. Value Health. 2006;9:193-8.

6. Fidler C, Elmelund CT, Gillard S. Hypoglycemia: an overview of fear of hypoglycemia, quality-of-life, and impact on costs. J Med Econ. 2011;14:646-55.

7. Frier BM. How hypoglycaemia can affect the life of a person with diabetes. Diabetes Metab Res Rev. 2008;24:87-92.
8. Leiter LA, Yale JF, Chiasson JL, Harris SB, Kleinstiver $P$, Sauriol L. Assessment of the impact of fear of hypoglycemic episodes on glycemic and hypoglycemic management. Can J Diabetes. 2005;29:186-92.

9. Seaquist ER, Anderson J, Childs B, et al. Hypoglycemia and diabetes: a report of a workgroup of the American Diabetes Association and the Endocrine Society. Diabetes Care. 2013;36:1384-95.

10. Hammer M, Lammert M, Mejias SM, Kern W, Frier BM. Costs of managing severe hypoglycaemia in three European countries. J Med Econ. 2009;12:281-90.

11. Leese GP, Wang J, Broomhall J, et al. Frequency of severe hypoglycemia requiring emergency treatment in type 1 and type 2 diabetes: a population-based study of health service resource use. Diabetes Care. 2003;26:1176-80.

12. McEwan P, Larsen TB, Wolden M, Jacobsen J, Evans M. Healthcare resource implications of hypoglycemia-related hospital admissions and inpatient hypoglycemia: retrospective record-linked cohort studies in England. BMJ Open Diabetes Res Care. 2015;3:e000057.

13. Chodick G, Heymann AD, Shalev V, Kookia E. The epidemiology of diabetes in a large Israeli HMO. Eur J Epidemiol. 2003;18:1143-6.

14. Heymann AD, Chodick G, Halkin H, et al. The implementation of managed care for diabetes using medical informatics in a large Preferred Provider Organization. Diabetes Res Clin Pract. 2006;71:290-8.

15. World Medical Association Declaration of Helsinki. Ethical Principles for Medical Research Involving Human Subjects. Presented at the 59th WMA General Assembly, Seoul, October 2008. http:// www.wma.net/en/30publications/10policies/b3/ 17c.pdf; 2008. Accessed 30 Mar 2016.

16. von Elm E, Altman DG, Egger M, Pocock SJ, Gøtzsche PC, Vandenbroucke JP, STROBE Initiative. The Strengthening the Reporting of Observational Studies in Epidemiology (STROBE) statement: guidelines for reporting observational studies. J Clin Epidemiol. 2008;61:344-9.

17. Heller SR, Frier BM, Herslov ML, Gundgaard J, Gough SC. Severe hypoglycaemia in adults with insulin-treated diabetes: impact on healthcare resources. Diabet Med. 2016;33:471-7.

18. Greco D, Pisciotta M, Gambina F, Maggio F. Severe hypoglycaemia leading to hospital admission in type 2 diabetic patients aged 80 years or older. Exp Clin Endocrinol Diabetes. 2010;118:215-9. 
19. Lipska KJ, Ross JS, Wang Y, et al. National trends in US hospital admissions for hyperglycemia and hypoglycemia among medicare beneficiaries, 1999 to 2011. JAMA Intern Med. 2014;174:1116-24.

20. Budnitz DS, Lovegrove MC, Shehab N, Richards CL. Emergency hospitalizations for adverse drug events in older Americans. N Engl J Med. 2011;365:2002-12.
21. Lammert M, Hammer M, Frier BM. Management of severe hypoglycaemia: cultural similarities, differences and resource consumption in three European countries. J Med Econ. 2009;12:269-80. 